Gut and Liver, Vol. 9, No. 6, November 2015, pp. 699-700

\title{
The Role of Barrier Dysfunction and Change of Claudin Expression in Inflammatory Bowel Disease
}

\author{
Tae II Kim \\ Department of Internal Medicine and Institute of Gastroenterology, Yonsei University College of Medicine, Seoul, Korea
}

See "Changes in the Expression and Distribution of Claudins, Increased Epithelial Apoptosis, and a Mannan-Binding LectinAssociated Immune Response Lead to Barrier Dysfunction in Dextran Sodium Sulfate-Induced Rat Colitis" by Bosi Yuan, et al. on page 734-740, Vol. 9. No. 6, 2015

The polarized intestinal epithelial layer has a critical function as a semipermeable barrier that separates the luminal contents from the subepithelial structure of intestine. The role of barrier dysfunction in intestinal epithelium has been known in many intestinal diseases. Many insults to epithelia such as ischemia/ reperfusion, inflammation, and toxic injury lead to the loss of this barrier function, which can be measured as increased intestinal permeability in vivo or decreased transepithelial electrical resistance (TER) in vitro. Especially, intestinal barrier dysfunction has been shown to be involved in pathogenesis and progression of inflammatory bowel disease (IBD). ${ }^{1,2}$ This barrier dysfunction in inflamed mucosa is in large part due to specific downregulation of key tight junction (TJ)-associated proteins such as claudin, occludin, and ZO-1 by proinflammatory cytokines. $^{3}$

As an important route of molecular movement across this barrier, paracellular transport occurs through the most apical contact point between adjacent epithelial cells, and is regulated by TJs, composed of occludin, claudins, tricellulin, and junctional adhesion molecules. ${ }^{4}$ Among these molecules, the 27 members of claudin family are expressed differentially in many tissues including intestinal tissue, and exhibit variable functional properties. The primary role of claudins in the TJ is the regulation of paracellular selectivity to small ions between individual epithelial cells. ${ }^{4}$ The majority of claudin isoforms act to increase TER, by selective decreases in cation permeability. ${ }^{4}$ In contrast to the other claudins, claudin 2 expression decreases "tightness" of the TJ in leaky epithelial tissues, by increasing small cation permeability, through cation-permeable pores. The combined expression patterns and ratios of the individual claudin family members determine the barrier function of tissuespecific TJs and therefore, the physiology of the tissue. ${ }^{4}$

Yuan et $a .^{5}$ showed the changes in expression and distribution of claudins, increased epithelial apoptosis, and decreased mannan-binding lectin (MBL)-associated immune response in ulcerative colitis (UC), and their relationships with barrier dysfunction. Although the animal model of dextran sulfate sodium colitis is not enough to explain whole aspects of epithelial barrier dysfunction of UC, they showed significant changes of major claudin molecules, such as downregulation and redistribution of sealing claudins (claudin 3, 5, and 8) and increased expression of the pore-forming claudin 2, which have been known in previous studies. ${ }^{2,6}$ They also showed additional relationship of apoptosis to barrier dysfunction. Apoptosis is well known factor of mucosal injury in many intestinal diseases, which can lead to barrier dysfunction. As for the relationship between decreased MBL-associated immune response and barrier dysfunction, there is no definite direct evidence in their direct interaction, and this relationship may be secondary consequence of inflammatory response in IBD. To support the direct causal relationship between MBL-associated specific immune response and barrier dysfunction, further studies are needed to show direct effect of MBLassociated immune reaction on barrier dysfunction. Actually, in many pathophysiologic results of intestinal epithelial changes in IBD, it is difficult to elucidate the causal relationship between barrier dysfunction and mucosal injury and inflammation. The barrier dysfunction can be involved in initiation and propagation of inflammation, and inflammatory cytokines can induce

Correspondence to: Tae Il Kim

Division of Gastroenterology, Department of Internal Medicine, Yonsei University College of Medicine, 50 Yonsei-ro, Seodaemun-gu, Seoul 03722, Korea

Tel: +82-2-2228-1965, Fax: +82-2-393-6884, E-mail: taeilkim@yuhs.ac

pISSN 1976-2283 eISSN 2005-1212 http://dx.doi.org/10.5009/gnl15430

@ This is an Open Access article distributed under the terms of the Creative Commons Attribution Non-Commercial License (http://creativecommons.org/licenses/by-nc/4.0) which permits unrestricted non-commercial use, distribution, and reproduction in any medium, provided the original work is properly cited. 
barrier dysfunction with vicious cycles.

Changes of barrier function can induce harmful or beneficial effects on intestinal mucosa, depending on intestinal milieu. Increased epithelial permeability may lead to epithelial injury by allowing inappropriate influx of molecules through the TJ, which may induce immune activation. In some conditions, growth factors moved through TJ can bind and activate their basolateral receptors, activating proliferative signaling pathway. ${ }^{7,8}$ In other way, increased TJ permeability in normal leaky epithelium can give a physiologic role to wash out molecules that may inappropriately diffuse from the intestinal lumen.

Moreover, many factors from microenvironment of intestine also are involved in regulation of barrier function. Some growth factors from epithelial cells and microenvironment like epidermal growth factor and keratinocyte growth factor, have a role in maintenance and change of barrier function via change of claudin expression. ${ }^{9}$ In pathogenesis, propagation, and mucosal recovery of IBD, many inflammatory cells and mesenchymal cells gathered to inflamed sites, and secreted a lot of factors like inflammatory cytokines and growth factors, for aggravation of inflammation and mucosal repair. In the aspects of barrier function, inflammatory cytokines and growth factors have different roles, such as decrease or increase of epithelial tightness. ${ }^{9,10}$ Therefore, epithelial barrier function will be involved in every steps of IBD, and their relationships will be complicated in the cause-and-effect relationship.

In addition, claudins, main molecules involved in regulation of barrier function, have been implicated in other pathophysiologic mechanisms besides barrier function. In such cases of epithelial damage such as IBD, claudin 2 expression increase in the progenitor compartment at the base of the crypts, a leaky epithelium, where the majority of cell proliferation occurs. ${ }^{4}$ These findings suggest that inflammatory damaged colon could induce more primitive proliferating crypt base cells to promote repair function via claudin 2 expression, resulting in a proliferative response. Moreover, Mankertz et al. ${ }^{11}$ has shown the presence of functional cross-talk between CDX2 and the Wnt pathway in the positive regulation of claudin 2 expression, indicating the possible role of Wnt pathway-related claudin 2 expression in progenitor cell area of crypt for epithelial repair. In this regard, increased claudin 2 expression might be related to activation of epithelial progenitor cells by stromal factors for rapid repair of damaged mucosa. In some studies, overexpression of claudin 2 induced more proliferative response and cell migratory activity, and claudin 2 knockdown blocked growth factor-induced proliferation. ${ }^{8}$ Collectively, these data suggest that increased claudin 2 expression plays a role in promoting cell proliferation and migration, which may augment epithelial repair processes in IBD. However, it remains to be answered how claudin 2, a charge-selective regulatory molecule of TJs, has a direct relationship to cell proliferation and migration.
In the future study, we need to investigate the detailed mechanisms of epithelial barrier dysfunction and permeability defects, and their role in IBD, and extend to the development of new therapeutic application, using modulation of epithelial barrier function.

\section{CONFLICTS OF INTEREST}

No potential conflict of interest relevant to this article was reported.

\section{REFERENCES}

1. Hollander D. Intestinal permeability, leaky gut, and intestinal disorders. Curr Gastroenterol Rep 1999;1:410-416.

2. Salim SY, Söderholm JD. Importance of disrupted intestinal barrier in inflammatory bowel diseases. Inflamm Bowel Dis 2011;17:362381

3. Nusrat A, Turner JR, Madara JL. Molecular physiology and pathophysiology of tight junctions. IV. Regulation of tight junctions by extracellular stimuli: nutrients, cytokines, and immune cells. Am J Physiol Gastrointest Liver Physiol 2000;279:G851-G857.

4. Krause G, Winkler L, Mueller SL, Haseloff RF, Piontek J, Blasig IE. Structure and function of claudins. Biochim Biophys Acta 2008; 1778:631-645.

5. Yuan B, Zhou S, Lu Y, et al. Changes in the expression and distribution of claudins, increased epithelial apoptosis, and a mannanbinding lectin-associated immune response lead to barrier dysfunction in dextran sodium sulfate-induced rat colitis. Gut Liver 2015;9:734-740.

6. Zeissig S, Bürgel N, Günzel D, et al. Changes in expression and distribution of claudin 2, 5 and 8 lead to discontinuous tight junctions and barrier dysfunction in active Crohn's disease. Gut 2007;56:6172.

7. Bücker R, Schumann M, Amasheh S, Schulzke JD. Claudins in intestinal function and disease. In: Yu AS, ed. Claudins. Volume 65. Amsterdam: Academic, 2010:195-227.

8. Dhawan P, Ahmad R, Chaturvedi R, et al. Claudin-2 expression increases tumorigenicity of colon cancer cells: role of epidermal growth factor receptor activation. Oncogene 2011;30:3234-3247.

9. Kim TI, Poulin EJ, Blask E, et al. Myofibroblast keratinocyte growth factor reduces tight junctional integrity and increases claudin-2 levels in polarized Caco-2 cells. Growth Factors 2012;30:320-332.

10. Lee HS, Kim DK, KimYB, Lee KJ. Effect of acute stress on immune cell counts and the expression of tight junction proteins in the duodenal mucosa of rats. Gut Liver 2013;7:190-196.

11. Mankertz J, Hillenbrand B, Tavalali S, Huber O, Fromm M, Schulzke JD. Functional crosstalk between Wnt signaling and Cdxrelated transcriptional activation in the regulation of the claudin-2 promoter activity. Biochem Biophys Res Commun 2004;314:10011007. 